\title{
Gasto público, permanencia en el poder y crecimiento económico
}

\author{
JUAN MARROQUÍN ARREOLA \\ Profesor de economía, ESCUELA SUPERIOR DE ECONOMÍA DEL INSTITUTO \\ POLITÉCNICO NACIONAL, MEXICO.E-mail: juanmarro@gmail.com
}

HUMBERTO RÍOS BOLÍVAR

Profesor de economía, ESCUELA SUPERIOR DE ECONOMÍA DEL INSTITUTO POLITÉCNICO NACIONAL, MEXICO.E-mail: hriosb@hotmail.com

\section{RESUMEN}

El objetivo de este documento es analizar cómo el gobierno determina la proporción de gasto público para maximizar su beneficio político y permanecer en el poder. Usando modelos de crecimiento y haciendo modificaciones en los mismos, este estudio demuestra que las acciones de gasto público y la tasa de crecimiento se determinan en función de los parámetros de ganancia política. Asimismo, los resultados de las simulaciones muestran que si la ganancia política de las políticas de distribuciónes alta, el gobierno destinará una mayor parte del fondo para fines de distribución que afectan negativamente al crecimiento económico.

Palabras clave: Gobierno egoísta, políticas distributivas, ganancia política, crecimiento económico.

\section{Public Spending, Staying Power and Economic Growth}

\begin{abstract}
The aim of this paper is to analyze how the government determines the proportion of public spending to maximize its political benefit and stay in power. Using growth models and making modifications in them, this study demonstrates that the actions of public expenditure and growth rate are determined by the parameters of political gain. Also, the results of the simulations show that if the political gain of distribution policy is high, the government will allocate a greater share of the fund for purposes of distribution adversely affecting economic growth.
\end{abstract}

Keywords: Government Selfish, Distributive Policies, Political Gain, Economic Growth.

Clasificación JEL: C02, H11, H53, O43

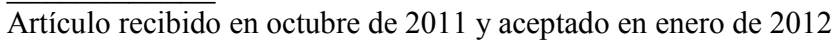

Artículo disponible en versión electrónica en la página www.revista-eea.net, ref. ə-30108 


\section{INTRODUCCIÓN}

El papel del gobierno en el desarrollo económico, el tamaño óptimo del gobierno, la naturaleza del gasto público y su impacto en el crecimiento, el gasto público y la corrupción, la política de distribución y el crecimiento económico se han convertido en temas importantes en la literatura de la política públicas y gestión fiscal (Alesina y Rodrik, 1994; Meltzer y Richard, 1981; Persson y Tabellini, 1994). A menudo se habla de política fiscal óptima, el gobierno benévolo y la maximización del crecimiento. Sin embargo, a veces la maximización del crecimiento no puede ser el objetivo principal del gobierno debido a que el gobierno puede estar más interesado en incrementar su beneficio político mediante la manipulación de instrumentos fiscales para permanecer en el poder.

El gobierno, especialmente en los países en desarrollo, muy a menudo estratégicamente adopta medidas a corto plazo populistas y recurre a las políticas distributivas sacrificando el crecimiento de largo plazo (Sasmal, 2004; Marjit, Kolmar y Mukherjee, 2001, Marjit y Maity, 2006). La estabilidad política puede muy bien coexistir con el estancamiento económico en una configuración democrática (Sarkar, 2006). Igualmente, los impuestos y gasto público no son el único mecanismo de transferencias de ingresos. Economía informal y gobernanza débil pueden ser una ruta alternativa a través del cual la redistribución del ingreso puede tener lugar (Marjit, Mukherjee y Kolmar, 2006). Marjit, Kolmar y Mukherjee (2001) muestran que cuanto mayor sea el alcance de la política de redistribución, peor será la calidad de la inversión pública.

La teoría del votante mediano dice que si la desigualdad es alta habrá una gran demanda de gobierno que afecta negativamente el crecimiento económico (Alesina y Rodrik, 1994). Pero no sólo los pobres son los que se favorecen de la distribución y beneficios directos e inmediatos del gobierno. Aidit y Shevets (2011) y Posner y Kramon (2011) mencionan que los grupos pudientes, como los grupos empresariales, los grandes agricultores, burócratas, empleados gubernamentales y otros grupos de interés también están muy interesados en extraer los máximos beneficios directos e inmediatos por parte del gobierno en forma de subvenciones, sueldos, dietas y otras concesiones. Por lo tanto, dada la preferencia de la gente y la coacción política de buscar apoyo de la mayoría en un conjunto democrático, el gobierno está más obligado a adoptar medidas populistas a corto plazo, que puede no ser útil para el crecimiento a largo plazo. Sin embargo, esta particular naturaleza de las decisiones fiscales y su impacto en el crecimiento no se tratan en la literatura, en ese sentido, especialmente en el contexto de los países en desarrollo. Contrariamente a la teoría del votante mediano, los trabajos de Banerjee y Newman (1993) y Galor y Zeira (1993) indican la posibilidad de un resultado diferente. El argumento es que si las políticas redistributivas pueden potenciar el capital humano, no se puede retrasar el crecimiento. 
Nuestro sentido común sugiere que la inversión a largo plazo en carreteras, ferrocarriles, aeropuertos, irrigación, electricidad, telecomunicaciones, educación, salud, ciencia y tecnología, y otras instalaciones de infraestructura aceleran el crecimiento económico, Alfonso y Sousa (2009) y Galor (2011) confirman este argumento. Sin embargo, algunos estudios empíricos muestran resultados mixtos. Devarajan et al. (1996) y Ghosh y Gregoriou (2008) encontraron que el gasto distributivo es más productivo que los gastos de capital, mientras que en Chen (2006), Cristiano et al. (2009), García-Peñalosa y Turnovsky (2006) el resultado es diferente. Los estudios sobre la base de datos entre países son demasiado agregados en la naturaleza y pasan por alto muchos factores locales. Por lo tanto, más estudios científicos son necesarios para capturar el efecto del gasto público sobre el crecimiento de una manera apropiada. Marjit, Mukherjee y Kolmar, (2006), mencionan que para que los efectos del gasto público se vean reflejados en el proceso de crecimiento no sólo depende de la composición del gasto público, sino también en la naturaleza del crecimiento, lapso de tiempo de completar los proyectos públicos y un mecanismo adecuado de transmisión de los efectos externos de los gastos en el crecimiento.

La investigación sobre el comportamiento del gobierno con respecto a las decisiones fiscales y su impacto en el crecimiento económico en el entorno democrático de los países en desarrollo son relativamente escasos. Marjit y Maity (2006) y Marjit, Mukherjee y Kolmar (2006) tienen algunas importantes contribuciones en este campo. Samal $(2002,2004,2007)$ encuentra que si la proporción de pobres es mayor en una sociedad, el gobierno va a recurrir a políticas más redistributivas, con sus implicaciones negativas para el crecimiento a largo plazo. El objetivo de este trabajo es examinar el impacto de las decisiones políticas del gobierno sobre el crecimiento económico en un marco endógeno con la estructura de la literatura existente y haciendo modificaciones en ella. El Modelo de Barro (1990) es modificado mediante la inserción de dos tipos de gasto público en la función de producción. Los dos tipos de gasto público son: inversión para el crecimiento a largo plazo (G1) y los gastos de la política distributiva (G2).

Asimismo, para demostrar cómo el gobierno determina la proporción de gasto público para maximizar su beneficio político y para examinar el efecto de las decisiones gubernamentales sobre el crecimiento económico, se realizan algunas modificaciones al modelo de Agenor (2008). Los resultados demuestran que si G2 genera un mayor rédito político, el gobierno se inclina a asignar más fondos a G2 que socava el crecimiento a largo plazo. La elección del modelo de Barro y Agenor se debe a que dichos modelos permiten estudiar de manera clara y sencilla como el gasto público se vincula con el crecimiento económico a través de la política distributiva, es decir, dada las características de los modelos, éstos permiten introducir parámetros de ganancia política y capacidad del 
gobierno para obtener ventaja política que son los parámetros que nos permiten analizar como la política distributiva se vincula con el crecimiento económico.

El documento se organiza de la siguiente manera: en la segunda sección, se presenta el marco teórico del crecimiento endógeno con gasto público. En la tercera sección, se presenta el esquema del modelo original de Barro. En la cuarta sección, se expone la modificación al modelo de Barro, así como la determinación de la tasa impositiva y la naturaleza del gasto público. En la quinta sección, se hace la simulación del modelo. Finalmente en al sexta sección, se exponen las conclusiones.

\section{CRECIMIENTO ENDÓGENO CON GASTO PÚBLICO}

La acumulación de capital, el crecimiento de la fuerza laboral y el progreso técnico se consideran generalmente como las tres principales fuerzas de crecimiento a largo plazo en un país. Si el crecimiento se produce debido a los avances técnicos que vienen de fuera del sistema de producción, como se ha explicado en los modelos de Harrod y Solow, ese se llama progreso técnico exógeno. Pero puede ser endógeno al sistema. Más específicamente, puede ser el resultado de algunas actividades con propósito de $\mathrm{I}+\mathrm{D}$ de un organismo privado o por el gobierno dentro del sistema. En el período posterior en la década de 1980, un grupo de teóricos del crecimiento liderado por Romer (1986, 1990), construyen una clase de modelos de crecimiento en el que los principales factores determinantes del crecimiento se determinan de manera endógena mediante la optimización de los agentes dentro del sistema. Cuando el crecimiento es determinado por factores determinados endógenamente en tales modelos, se llama crecimiento endógeno (Barro y Sala-i-Martin, 1995). En los modelos de Harrod y Solow, la tasa de ahorro es exógena. Pero en el modelo de Ramsey, las tasas de ahorro y formación de capital se determinan mediante la optimización del comportamiento de los hogares. La tasa óptima de ahorro y acumulación de capital (Ramsery, 1928), crecimiento de la población (Barro y Becker, 1989), acumulación de conocimiento y capital humano (Romer, 1986, 1990; Uzawa, 1965), asignación óptima de recursos a la I+D para el progreso técnico (Grossman y Helpman, 1991) son las principales obras de la teoría del crecimiento endógeno. Los modelos de crecimiento endógeno no solamente determinan endógenamente los factores clave del crecimiento, también proveen un mecanismo en el cual la interacción del comportamiento de la optimización de los agentes en el sistema determina la trayectoria óptima de los determinantes clave del crecimiento así como la trayectoria del crecimiento en el tiempo.

Un importante requisito del crecimiento endógeno es la eliminación de los rendimientos decrecientes del capital. El problema con el modelo de Solow es que si el capital por unidad de trabajo $(\mathrm{K} / \mathrm{L})$ rescrito como $\mathrm{k}$ aumenta, el producto marginal del capital disminuye (condiciones de Inada). Pero si los rendi- 
mientos decrecientes pueden ser detenidos, el crecimiento puede ser perpetuo y este puede ser hecho por el crecimiento continuo del capital humano, conocimiento y progreso técnico mediante la necesaria inversión en actividades de investigación y desarrollo en un marco endógeno. La idea de la no disminución de la rentabilidad del capital es bastante plausible si pensamos en una perspectiva más amplia para incluir el capital humano. El modelo de crecimiento endógeno se puede construir tanto en el contexto de rendimientos constantes y crecientes del capital. El modelo simple en esta categoría con rendimientos constantes al capital es el modelo AK (Barro y Sala-i-Martin, 1995). El modelo con rendimientos crecientes al capital en un marco endógeno fue desarrollado por Romer (1986) y Rebelo (1991). El gobierno puede acelerar el crecimiento jugando un papel importante en el desarrollo de infraestructura, capital humano, conocimiento y tecnología. Barro (1990), Alesina y Rodrick (1994) y Barro y Sala-i-Martin explicaron como el gasto público tanto en la producción y consumo afectan el crecimiento de largo plazo. Obviamente, la tasa de impuestos, la asignación de fondos para inversiones productivas y la política distributiva, así como la corrupción en el nivel de gobierno, se han convertido en temas importante en el proceso de crecimiento.

Como se dijo antes, en este estudio se considera un modelo donde el gobierno distribuye sus fondos en dos formas; (a) Los gastos de desarrollo a largo plazo (G1) y (b) el gasto en la política distributiva (G2). Sea asume que los dos tipos de gasto elevan la productividad, pero $(\mathrm{G} 1)$ es más productivo que $(\mathrm{G} 2)^{1}$. El modelo muestra que si el gobierno tiene que asignar una mayor proporción de los fondos de (G2) para cumplir con sus políticas compulsivas, la tasa de crecimiento será menor. (ii) La pérdida de fondos debido a la corrupción reduce la tasa de crecimiento.

\section{ESQUEMA DEL MODELO DE BARRO}

El crecimiento endógeno con gasto público tal como se describe en el modelo de Barro y Sala-i-Martin, tiene especificada una función de producción CobbDouglas:

$$
Y=A K^{\alpha} L^{1-\alpha} G^{1-\alpha}
$$

Donde $G$ es un bien público productivo financiado por la misma cantidad de la recaudación de impuestos, $Y$ es el producto, $K$ es el capital privado, $L$ es el trabajo y $A$ es un término constante. $G$ es la compra total del gobierno de la

\footnotetext{
${ }^{1}$ Cuando hablamos de que el gasto distributivo es menos productivo y reduce el crecimiento, nos estamos basando en el estudio de Marjit, Kolmar y Mukherjee (2001), donde muestran que si no existe gasto en infraestructura para el desarrollo, entonces no se acelerará el crecimiento económico, por lo tanto, cuanto mayor sea el alcance de la política de redistribución, peor será la calidad de la inversión pública.
} 
producción privada que se utiliza para el desarrollo de la infraestructura física, capital humano, la ley y el orden, etc.

Ahora, la ecuación (1) se puede escribir como sigue:

$$
Y=A K^{\alpha}(L G)^{1-\alpha}
$$

Suponiendo que $L$ es constante, si $G$ se mantiene sin cambios, la función tiene rendimientos decrecientes en $K$. Pero si $G$ aumenta a la misma tasa que $K$, la función tiene rendimientos constantes en $K$ y $G$, y como resultado, la economía se vuelve capaz de tener un crecimiento endógeno.

Aquí, el gobierno sigue una política de presupuesto equilibrado y una tasa de impuesto proporcional, es decir:

$$
G=r Y
$$

Donde $r$ es la tasa de impuesto. Se supone que la tasa de impuesto $(r)$ y el gasto público - proporción del ingreso $(G / Y)$ - son constantes en el tiempo.

Las condiciones de maximización de beneficios de las empresas requieren que (i) la tasa de salario $(W)$ sea igual al producto marginal del trabajo después de impuestos, y (ii) la tasa de alquiler $(R)$ sea igual al producto marginal del capital después de impuestos. Esto es,

$$
R=(1-r) . \delta Y / \delta K=(1-r) \alpha A k^{-(1-\alpha)} G^{1-\alpha}
$$

Solucionando $G$ de la función de producción (1) y sustituyendo el valor en (4), tenemos

$$
R=(1-r) \alpha A^{\alpha}(L r)^{(1-\alpha) / \alpha}
$$

$R$ es ahora independiente de $k$, y dado los valores de $A, L, r, \alpha$, la tasa de alquiler $(R)$ es constante, i.e. productividad marginal del capital $\left(P M_{K}\right)$ es constante. Por lo que el requisito de crecimiento endógeno se cumple.

\section{MODIFICACIÓN DEL MODELO DE BARRO}

En el modelo básico de Barro, el gobierno gasta la totalidad del importe de la recaudación de impuestos en el servicio productivo. En una extensión del modelo, se considera la situación donde el gobierno gasta una parte de los ingresos fiscales para el consumo de los hogares y para el propio gobierno. En este caso, se encuentra que la tasa de crecimiento en el tiempo es menor de lo que habría sido si el total de los ingresos de impuestos se usaran para propuestas productivas.

La modificación del modelo también supone una política de presupuesto equilibrado. Sin embargo, los ingresos de impuestos se gastan en dos formas: (i) los gastos de desarrollo a largo plazo $\left(G_{1}\right)$ y (ii) el gasto en políticas distribu- 
tivas $\left(G_{2}\right)$. Chen (2006) considera los gastos de producción y consumo en el presupuesto. Como en Devarajan et al. (1996) y Ghosh y Gregoriou (2008), en la presente modificación $G_{1}$ y $G_{2}$ también son productivos, pero $G_{2}$ se supone que es menos productivo que $\mathrm{G}_{1}$ (ver nota 1 ). En este caso, aquí, el gasto púbico en la política de redistribución incluye una amplia variedad de gastos que incluye las transferencias directas y subsidios a los pobres. Esto se hace para cumplir con las compulsiones electorales del gobierno. Es importante señalar que estos gastos no son totalmente improductivos debido a que se basa en la hipótesis de que estos gastos mejoran la productividad a través de la eficiencia nutricional, la inteligencia humana, mayores incentivos y la participación en el proceso de producción. Sin embargo, su impacto en la producción es menor que la inversión a largo plazo.

Se ha considerado una función de producción Cobb-Douglas en la que $G_{1}$ y $G_{2}$ se incluyen como factores productivos, además del capital privado y laboral, es decir,

$$
Y=A K^{\alpha} G_{1}\left(L G_{2}\right)^{1-\alpha-\beta}
$$

Donde $K$ es el capital privado, $L$ es el trabajo y $Y$ es el producto. Asimismo,

$$
\left.\frac{\partial Y}{\partial G_{1}}\right\rangle \frac{\partial Y}{\partial G_{2}}
$$

se supone que $L$ es constante como antes. $L$ se mantiene constante si $G_{1}$ y $G_{2}$ aumentan a la misma tasa que $K$, entonces habrá rendimientos constantes a escala en la producción. $L$ y $G_{2}$ son sustitutos perfectos. De hecho, aquí el trabajo se mide en términos de eficiencia y se supone que $\beta>1-\alpha-\beta$.

Puesto que el gobierno sigue una política de equilibrio presupuestario, la recaudación total de impuestos $(T)$ es igual al gasto público total $(G)$, i.e. $T=$ $r . Y=G=G_{1}+G_{2}$.

Suponiendo que $r, \lambda$ y $\psi$ son la tasa de impuesto, la parte del gasto público para inversión a largo plazo y la proporción de fugas de fondos del gobierno debido a la corrupción están dadas y son constantes en el tiempo.

Así, el fondo neto disponible con el gobierno es:

$$
\lambda(1-\psi) \cdot r . Y+(1-\lambda)(1-\psi) \cdot r . Y=(1-\psi) \cdot r . Y
$$

La ecuación (5) se puede utilizar para incorporar la política de gobierno en el modelo de crecimiento a través de la ecuación (7).

Ahora para encontrar $P M_{K}$, se considera la función de producción en la ecuación (1):

$$
Y=A K^{\alpha} G_{1}^{\beta}\left(L G_{2}\right)^{1-\alpha-\beta}
$$


En términos per cápita se reduce a

$$
y=A k^{\alpha} g_{1}^{\beta}\left(G_{2}\right)^{1-\alpha-\beta}
$$

$P M_{K}$ se puede derivar de la ecuación (8) de la siguiente manera

$$
P M_{K}=\frac{\partial Y}{\partial K}=L \cdot A \cdot g_{1}^{\beta} G_{2}^{1-\alpha-\beta} \alpha k^{\alpha-1} \cdot \frac{\partial k}{\partial K}=A g_{1}^{\beta} G_{2}^{1-\alpha-\beta} \alpha k^{-(1-\alpha)}
$$

De la ecuación (7) se puede escribir

$$
g_{1}=\frac{G_{1}}{L}=\frac{1}{L} \cdot \lambda(1-\psi) r . Y
$$

$\mathrm{y}$

$$
G_{2}=(1-\lambda)(1-\psi) Y
$$

Luego, sustituyendo el valor de $g_{l}$ y $G_{2}$ en (9) se obtiene

$$
P M_{K}=\alpha A^{\frac{1}{\alpha}} \lambda^{\frac{\beta}{\alpha}}(1-\lambda)^{\frac{1-\alpha-\beta}{\alpha}}[(1-\psi) r]^{\frac{1-\alpha}{\alpha}} L^{\frac{\beta}{\alpha}}
$$

(Ver apendice)

Aquí también, $\mathrm{PM}_{\mathrm{K}}$ es independiente de $k \mathrm{y}$ es constante dado los parámetros y dados los valores de $r, \lambda$ y $\psi$. Se supone que $\mathrm{PM}_{\mathrm{K}}$ depende de los parámetros tecnológicos y los valores dados de $\lambda, \psi$ y $r$.

Ahora, al igualar la tasa de alquiler $(R)$ con $P M_{K}$ después de impuestos y usando la regla de ahorro de Ramsey, tenemos

$$
g_{k}=g_{y}=g_{c}=\frac{1}{\theta}\left\{(1-r) \alpha A^{\frac{1}{\alpha}} \lambda^{\frac{\beta}{\alpha}}(1-\lambda)^{\frac{1-\alpha-\beta}{\alpha}}[(1-\psi) r]^{\frac{1-\alpha}{\alpha}} L^{\frac{\beta}{\alpha}}-\rho\right\}
$$

en un crecimiento de estado estacionario.

La ecuación (11) tiene importantes implicaciones. A medida que la tasa de crecimiento incorpora elementos de la corrupción y la asignación de fondos para la política distributiva, la tasa de crecimiento es más baja. Dado los valores de $r$ y $\psi$, y los parámetros del sistema, si el desvío de fondos de políticas distributivas para inversión en el largo plazo mejorara el crecimiento, éste dependerá de los valores de $\lambda$ y las elasticidades de producción de $K, G_{I}$ y $G_{2}$.

Derivando (11) con respecto a $\lambda$, se obtiene 


$$
\begin{array}{ccc}
\frac{\partial g_{y}}{\partial \lambda}=0 & \text { si } & \lambda=\frac{\beta}{1-\alpha} \\
\langle & & >
\end{array}
$$

Por el supuesto en la función de producción

$$
\beta>1-\alpha-\beta \text { i.e. } \frac{\beta}{1-\alpha}>\frac{1}{2}
$$

Esto implica que el incremento en $\lambda$ conducirá a un mayor crecimiento si $\lambda<$ $\frac{\beta}{1-\alpha}>\frac{1}{2}$. La tasa de crecimiento es máxima si $\lambda=\frac{\beta}{1-\alpha}$. Si la maximización del crecimiento es el objetivo, los fondos disponibles se distribuirán entre $G_{1}$ y $G_{2}$ hasta que el producto marginal de $G_{1}$ y $G_{2}$ sean iguales, y de esa igualdad tenemos que $\frac{G_{1}}{G_{2}}=\frac{\beta}{1-\alpha-\beta}>1$ (por nuestro supuesto de $\beta>1-\alpha-\beta)$.

Entonces, esto es consistente con $\lambda=\frac{\beta}{1-\beta}>\frac{1}{2}$. Eso significa que, para la maximización del crecimiento, se requiere que $\lambda>\frac{1}{2}$.

Si el gobierno es egoísta y su objetivo es la maximización de la ganancia política, puede elegir un valor de $\lambda$ menor que $\frac{\beta}{1-\alpha}$. La tasa de impuestos $(r)$ es otro determinante de la tasa de crecimiento. En Barro y Sala-i-Martin, el gobierno benevolente fija la tasa de impuesto en $r=1-\alpha$ para maximizar el crecimiento. Aquí en el modelo modificado, con el fin de maximizar la tasa de crecimiento, se deriva la ecuación (11) con respecto a $r$ y se iguala a cero, i.e. $\frac{\partial g_{y}}{\partial r}=0$. Después de la simplificación se obtiene $r=1-\alpha$. Esto significa que, la tasa de impuesto óptimo es igual a la suma de las elasticidades de producción de $\mathrm{G}_{1} \mathrm{y}_{2}$, i.e. $\beta+(1-\alpha-\beta)=1-\alpha$.

Esto implica que el crecimiento que maximiza la tasa impositiva es el mismo como en el modelo original incluso cuando el ingreso fiscal es gastado de dos formas diferentes y sus elasticidades de producción son diferentes. Sin embargo, si el objetivo del gobierno es la maximización de la ganancia política, el valor de $r$ puede ser diferente de $(1-\alpha)$. 


\subsection{Determinación de la tasa de impositiva óptima y composición del gasto público}

En la sección anterior se determinó la tasa de crecimiento suponiendo que la tasa de impuestos y la participación de los gastos públicos están dados y son constantes en el tiempo. Ghosh y Gregoriu (2008), en una extensión del modelo de Devarajan et al. (1996), construyen un modelo para determinar la tasa de impuesto óptimo y la proporción de capital y gasto corriente en un marco endógeno para maximizar el crecimiento. En Ghosh y Gregoriou, la política fiscal que maximiza el bienestar del gobierno benevolente, elige los instrumentos fiscales óptimos para maximizar la utilidad del agente representativo tomando las elecciones individuales en un marco descentralizado con una restricción dada. Ellos consideran una tecnología de producción CES con dos tipos de gasto público como en Devarajan et al. (1996):

$$
y=\left[\alpha k^{-\varepsilon}+\beta g_{1}^{-\varepsilon}+\gamma g_{2}^{-\varepsilon}\right]^{-\frac{1}{\varepsilon}}
$$

Donde $\alpha+\beta+\gamma=1, \varepsilon \geq-1$

La restricción presupuestaria del gobierno es: $g_{1}+g_{2}=\tau y$, donde $\tau$ es la tasa de impuesto.

Las preferencias del agente representativo están representadas por la elasticidad de sustitución constante en la utilidad como $U=\int_{0}^{\infty}\left(\frac{C^{1-\delta}-1}{1-\delta}\right) e^{-\rho t} d t$, donde la utilidad se deriva del consumo privado.

La proporción del gasto público son $g_{1}=\phi \mathfrak{y}$ y $g_{2}=(1-\phi) \succsim y$

La restricción presupuestaria del agente es: $\dot{k}=(1-\tau) y-C$

El agente representativo maximiza " $U$ " óptimamente eligiendo $C$ y $\dot{k}, \mathrm{y}$ deriva la trayectoria de crecimiento como

$$
\lambda=\frac{C}{C}=(1-\tau) \frac{\partial y}{\partial k}-\rho
$$

El gobierno benévolo determina óptimamente $\tau, g_{1}, g_{2}$ para maximizar el crecimiento tomando (11.1) y otras restricciones como dadas y el valor óptimo de $\tau, \phi$ y $(1-\phi)$ se determinan en función de los parámetros tecnológicos y de comportamiento. La implicación de este resultado es que aquí también los valores de la tasa de impuesto y proporción de gasto son constantes como en la sección anterior a pesar de que se determina endógenamente. La tasa de crecimiento también es determinada en función de los parámetros y si un cambio en 
la composición del gasto público llevara a un cambio en la tasa de crecimiento dependerá de estos parámetros.

\subsection{Determinación de las variables fiscales para maximización de la ganancia política}

Ahora se verá como las variables fiscales son determinadas por el gobierno cuando su objetivo es maximizar la ganancia política para mantener el poder mediante la manipulación de los instrumentos fiscales. Los dos tipos de gasto público son denotados por $\mathrm{G}_{1}$ y $\mathrm{G}_{2}$, respectivamente y se supone sistema fiscal de presupuesto equilibrado. Por lo tanto, $G_{1}+G_{2}=T=r$. $Y$ donde $Y$ es el ingreso, $r$ es la tasa de impuesto y $T$ es el total de ingresos fiscales. $\lambda$ es la proporción de ingresos fiscales asignados a $G_{1}$ y $(1-\lambda)$ es la proporción de $G_{2}$. Por lo tanto, tenemos

$$
G_{1}=\lambda r Y, \quad G_{2}=(1-\lambda) r Y
$$

En una democracia multipartidaria de bajos ingresos, el gobierno se ocupa básicamente de la ganancia a corto plazo y los partidos políticos no son muy diferentes unos de otros con respecto a sus objetivos y programas económicos. Los agentes privados obtienen utilidad del consumo privado $(C)$ y sus gastos de distribución $\left(G_{2}\right)$ en una forma no separable. Tanto $G_{1}$ y $G_{2}$ afectan la utilidad de los individuos a través de la producción. Puesto que $G_{2}$ involucra muchas medidas populistas y de distribución, y la oportunidad de la búsqueda de rentas está allí, el partido en el poder puede obtener algún rédito político de $G_{2}$. Por lo tanto, el gobierno como agente optimizador manipula los instrumentos fiscales para maximizar su ganancia política total, la cual incluye tanto la utilidad privada así como el interés propio del gobierno. Naturalmente, el gobierno puede asignar más fondos a $G_{2}$ a pesar de saber que puede afectar negativamente el crecimiento económico. Siguiendo a Agenor (2008), se considera una función de utilidad no separable en $C$ y $G_{2}$ para el agente privado como $U=\frac{\left(C G_{2}^{\varphi}\right)^{1-\theta}}{1-\theta}$ donde $\theta=\frac{1}{\sigma}$ es el reciproco de la elasticidad de sustitución intertemporal en la utilidad y $\varphi>0$ es la medida del valor asignado a la contribución de $G_{2}$ en la utilidad privada. A fin de garantizar la propiedad de concavidad de la función, las restricciones impuestas son: $\varphi(1-\theta)<1$ y $(1+\varphi)(1-\theta)<1$.

Como en Chen (2005), la función de utilidad puede ser escrita en una forma Cobb-Douglas

$$
U=\left\lfloor\left(C^{\alpha} G_{2}^{1-\alpha}\right)^{1-\theta}-1\right] / 1-\theta
$$


Puesto que $G_{2}$ genera algunos beneficios electorales para el gobierno a parte de contribuir a la utilidad privada, un término adicional se introduce en la función objetivo de Agenor (2008) para capturar la ganancia política en la toma de decisiones.

El propio interés del gobierno de $G_{2}$ se puede expresar como $\phi=\eta G_{2}^{\mu}$

Donde $\phi$ es la ganancia política, $\mu$ es la medida del valor asignado a la contribución de $G_{2}$ en ganancia política y $\eta$ es el parámetro que refleja la capacidad del gobierno para obtener la ventaja política de $G_{2}$. En este caso, $\eta>0,0$ $<\mu<1$.

La tecnología de producción Cobb-Douglas es de la forma:

$$
Y=A K^{\alpha} G_{1}^{\beta}\left(L G_{2}\right)^{1-\alpha-\beta}
$$

Se puede expresar como

$$
Y=\bar{A} K \lambda^{\beta / \alpha}(1-\lambda)^{\frac{1-\alpha-\beta}{\alpha}} \cdot r^{\frac{1-\alpha}{\alpha}}
$$

Donde $\bar{A}=A^{\frac{1}{\alpha}} L^{\frac{1-\alpha-\beta}{\alpha}}$ y $L$ es constante.

Del mismo modo, $G_{2}$ se puede expresar como

$$
G_{2}=r^{\frac{1}{\alpha}} \bar{A} K \lambda^{\frac{\beta}{\alpha}}(1-\lambda)^{\frac{1-\alpha-\beta}{\alpha}}
$$

El presupuesto de la economía es:

$$
Y=C+\dot{K}+G_{1}+G_{2} \text { ó } \dot{K}=(1-r) Y-C
$$

Teniendo en cuenta los valores de $r, \lambda$ y otros parámetros, el agente privado representativo maximiza

$$
V=\int_{0}^{T}\left\{\frac{\left(C G_{2}^{\varphi}\right)^{1-\theta}}{1-\theta}\right\} e^{-\rho t} \cdot d t \text {. Sujeto a } \dot{K}=(1-r) Y-C
$$

Este es un problema de optimización de periodo definido. En Agenor (2008) el agente privado resuelve el problema de optimización eligiendo $C$ y obteniendo la trayectoria del crecimiento para el consumo en un marco descentralizado como

$$
\frac{\dot{C}}{C}=-\sigma\left(\frac{\dot{v}}{v}\right)+\gamma\left(\frac{\dot{G}_{2}}{G_{2}}\right) \text { donde } \gamma=\sigma \varphi\left(1-\frac{1}{\sigma}\right)
$$


Y $v$ es el precio sombra actual de $K$. Después de la solución y la simplificación, la trayectoria del crecimiento se expresa en términos de los parámetros como

$$
\frac{\dot{C}}{C}=-\left(\frac{\sigma}{1-\gamma}\right)\{\rho-(1-r)(1-\alpha-\beta)\}\left[r^{\frac{1-\alpha}{\alpha}} \lambda^{\frac{\beta(1-\alpha)}{\alpha}}(1-\lambda)^{\frac{1-\alpha-\beta(1-\alpha)}{\alpha}}\right]
$$

La tasa de crecimiento del consumo depende de los valores dados de $r, \lambda$ y los parámetros.

En la modificación del modelo, se incorpora el propio interés del gobierno de $G_{2}$ en la función de ganancia política. Ahora el objetivo del gobierno se convierte en

$$
\operatorname{Max} W=\int_{0}^{T}\left[\frac{\left(C G_{2}^{\varphi}\right)^{1-\theta}}{1-\theta}+\eta G_{2}^{\mu}\right] e^{-\rho t} d t
$$

Sujeto a (14) y (16). Esto se resolverá por la elección óptima de $r$ y $\lambda$ sujeto a las restricciones.

Para la solución del problema de optimización dinámica (17), el Hamiltoniano es:

$$
H=\frac{\left(C G_{2}^{\varphi}\right)^{1-\theta}}{1-\theta}+\eta G_{2}^{\mu}+v[(1-r) Y-C]
$$

Aquí, $Y, G_{1}$ y $G_{2}$ depende de $r$ y $\lambda$ los cuales serán óptimamente determinados por el gobierno en términos de los parámetros [ver (12), (13)]. Esto significa que, las variables de control son $r$ y $\lambda$.

La condiciones de primer orden $\frac{\partial H}{\partial r}=0, \frac{\partial H}{\partial \lambda}=0$ dará el valor óptimo de $r$ y $\lambda$ en cada punto del tiempo. Se determinarán en términos de la variable de estado $K$, de $v$. Dos nuevos parámetros $\mu$ y $\eta$ entran en el juego de parámetros del modelo modificado.

Ahora, $\dot{K}$ y $\dot{v}$ junto con las condiciones de transversalidad trazan el camino de solución dinámica a este problema. A lo largo de la trayectoria óptima las variables de control $r$ y $\lambda$ se determinan en términos de los parámetros. El conjunto de parámetros es $\{\alpha, \beta, \theta, \varphi, \mu, \eta, \rho\}$.

La solución dinámica de este problema está dado por las siguientes dos ecuaciones diferenciales junto con las condiciones iniciales y de transversalidad: 


$$
\begin{aligned}
& \dot{K}=(1-r)\left\{\bar{A} K \lambda^{\beta / \alpha}(1-\lambda)^{\frac{1-\alpha-\beta}{\alpha}} r^{\frac{1-\alpha}{\alpha}}\right\}-C \\
& \dot{v}=-\frac{\partial H}{\partial K}+\rho v
\end{aligned}
$$

Suponiendo además que la solución es convergente y dinámicamente estable, la tasa de crecimiento de $\mathrm{K}$ se puede expresar como

$$
\frac{\dot{K}}{K}=(1-r)\left\{\bar{A} \lambda^{\beta / \alpha}(1-\lambda)^{\frac{1-\alpha-\beta}{\alpha}} r^{\frac{1-\alpha}{\alpha}}\right\}-\frac{C}{K}
$$

En el estado de equilibrio, todas las variables crecen a tasas constantes. Por lo tanto, la tasa de crecimiento es $g=\frac{C}{C}=\frac{K}{K}=\frac{Y}{Y}$. Esto significa que, $\left(\frac{C}{K}\right)$ es constante en el largo plazo y la tasa de crecimiento también es constante. La tasa de crecimiento se determina en términos de los parámetros. El rango de valores de $\mu$ у $\eta$, y su impacto en el crecimiento son de especial interés en este documento. Por lo tanto, nos gustaría ver que sucede en la tasa de crecimiento de $K$ en (21) si se producen cambios en los valores de $\mu$ y $\eta$. De acuerdo a la literatura del ciclo económico, calibramos los parámetros del modelo para obtener hechos empíricos a largo plazo simulando a una economía subdesarrollada y multipartidista como la economía mexicana dada sus condiciones económicas. Siguiendo a Cooley y Prescott (1995) el consumo representa el doble de la inversión en economías subdesarrolladas. El valor de capital se elige para garantizar el equilibrio plausible en el modelo. Asimismo, siguiendo a Futagami et al. (1993) $\alpha=0.3$ y $\beta=0.6$ suponen que los efectos del stock de capital son menos productivos que los del trabajo en economías subdesarrolladas, por lo que asumimos dichos valores dadas la condiciones de la economía mexicana. Los valores de $\mu$ y $\eta$ representan el porcentaje de gasto para obtener la ventaja política para poder permanecer en el poder. El interés que se toma como referencia es la tasa de cetes del banco de México la cual es de $8 \%$. Si los valores de $\mu$ у $\eta$ son tales que la ganancia política de $\mathrm{G}_{2}$ es muy alta, entonces el gobierno puede decidir asignar una mayor parte de los fondos para políticas distributivas socavando el crecimiento. 


\section{EFECTOS DE LOS CAMBIOS DE M Y H SOBRE EL CRECIMIENTO: UN ANÁLISIS NUMÉRICO}

Para obtener datos numéricos de la economía mexicana y conocer los efectos de los cambios en los instrumentos de ganancia política realizamos un análisis numérico.

Comenzamos la calibración utilizando un conjunto de parámetros representativos, los cuales son los siguientes:

$$
\begin{aligned}
& \alpha=0.3 \quad \beta=0.6 \quad \mu=0.2 \quad \eta=0.25 \quad \bar{A}=100 \quad K(0)=10000 \\
& C / K=2 \quad r=0.08 \quad \lambda=\mu+\eta
\end{aligned}
$$

Los resultados de la simulación se muestran en los Cuadros 1, 2 y 3.

\section{Cuadro 1}

\begin{tabular}{|c|c|c|c|}
\hline [en $\mu=0,1]$ & $k / k=$ & $\mathrm{DK} / \mathrm{K}=$ & 1.6156 \\
\hline [en $\mu=0,15$ ] & & $\mathrm{DK} / \mathrm{K}=$ & 1.5112 \\
\hline [en $\mu=0,20$ ] & & $\mathrm{DK} / \mathrm{K}=$ & 1.3990 \\
\hline [en $\mu=0,25$ ] & & $\mathrm{DK} / \mathrm{K}=$ & 1.2813 \\
\hline [en $\mu=0,3$ ] & & $\mathrm{DK} / \mathrm{K}=$ & 1.1603 \\
\hline [en $\mu=0,4$ ] & & $\mathrm{DK} / \mathrm{K}=$ & 0.9215 \\
\hline \multicolumn{4}{|l|}{ Con $\mu=0.2$} \\
\hline [en $\mu=0,1$ ] & $K / k=$ & $\mathrm{DK} / \mathrm{K}=$ & 1.7105 \\
\hline [en $\mu=0,15$ ] & & $\mathrm{DK} / \mathrm{K}=$ & 1.6156 \\
\hline [en $\mu=0,20$ ] & & $\mathrm{DK} / \mathrm{K}=$ & 1.5112 \\
\hline [en $\mu=0,25$ ] & & $\mathrm{DK} / \mathrm{K}=$ & 1.3990 \\
\hline [en $\mu=0,3$ ] & & $\mathrm{DK} / \mathrm{K}=$ & 1.2813 \\
\hline [en $\mu=0,4$ ] & & $\mathrm{DK} / \mathrm{K}=$ & 0.0392 \\
\hline
\end{tabular}

Resultados del cambio de $\mu$ y $\eta$ en $\mathrm{K}$ a través de $\lambda$

Fuente: Elaboración en base a resultados de la simulación. 


\section{Cuadro 2}

Resultados del $\mu$ y $\eta$ en $\mathrm{K}$ a través de $\mathrm{r}$

\begin{tabular}{|c|c|c|c|}
\hline [en $\mu=0,1, \eta=0.31$ ] & $k / k=$ & $\mathrm{DK} / \mathrm{K}=$ & 1.660 \\
\hline [en $\mu=0,11]$ & & $\mathrm{DK} / \mathrm{K}=$ & 1.683 \\
\hline [en $\mu=0,12$ ] & & $\mathrm{DK} / \mathrm{K}=$ & 1.707 \\
\hline [en $\mu=0,13$ ] & & $\mathrm{DK} / \mathrm{K}=$ & 1.731 \\
\hline [en $\mu=0,14$ ] & & $\mathrm{DK} / \mathrm{K}=$ & 1.756 \\
\hline [en $\eta=0.31, \mu=0,1]$ & $K / k=$ & $\mathrm{DK} / \mathrm{K}=$ & 1.660 \\
\hline [en $\mu=0,32$ ] & & $\mathrm{DK} / \mathrm{K}=$ & 1.605 \\
\hline [en $\mu=0,33$ ] & & $\mathrm{DK} / \mathrm{K}=$ & 1.543 \\
\hline [en $\mu=0,34]$ & & $\mathrm{DK} / \mathrm{K}=$ & 1.474 \\
\hline [en $\mu=0,35$ ] & & $\mathrm{DK} / \mathrm{K}=$ & 1.399 \\
\hline
\end{tabular}

Fuente: Elaboración en base a resultados de la simulación.

\section{Cuadro 3}

Efectos del cambio de valor de $\mu$ y $\eta$ sobre $\dot{K} / K$ a través de $\lambda$ y $\mathrm{r}$

\begin{tabular}{|c|c|c|c|}
\hline [en $\eta=0.3, \mu=0.1]$ & $k / k=$ & $\mathrm{DK} / \mathrm{K}=$ & 1.710 \\
\hline [en $\eta=0.31, \mu=0.1$ ] & & $\mathrm{DK} / \mathrm{K}=$ & 1.660 \\
\hline [en $\eta=0.32, \mu=0.1]$ & & $\mathrm{DK} / \mathrm{K}=$ & 1.605 \\
\hline [en $\eta=0.33, \mu=0.1$ ] & & $\mathrm{DK} / \mathrm{K}=$ & 1.543 \\
\hline [en $\eta=0.34, \mu=0.1$ ] & & $\mathrm{DK} / \mathrm{K}=$ & 1.474 \\
\hline
\end{tabular}

Fuente: Elaboración en base a resultados de la simulación.

Los resultados de la simulación muestran que el valor $\dot{K} / K$ tiende a disminuir a medida que $\mu$ y $\eta$ se elevan casi en todos los casos. Esto significa que la tasa de crecimiento disminuye a una tasa constante mientras la ganancia política $\mu$ y $\eta$ se eleva, tanto en el caso de mantener $\eta$ constante como en el caso contrario. Sin embargo, los efectos del cambio de $\mu$ a través de $r$ en el Cuadro 2 da diferentes resultados. En el primer caso, la tasa de crecimiento tiende a aumentar en lugar de disminuir, la explicación intuitiva puede ser que el efecto de un cambio de $\lambda$ en el crecimiento es mas directo dada la elasticidad de la producción de $G_{1}$ y $G_{2}$, pero en caso de aumentar la capacidad del gobierno de obtener ventaja política, la tasa de crecimiento tiende a disminuir. Puesto que no se obtiene una solución cerrada, $\lambda$ y $r$ se expresan en términos de una 
combinación lineal de $\mu$ y $\eta$. También se puede observar que en el Cuadro 3, los valores de la tasa de crecimiento de $K$ disminuyen mientras $\eta$ se eleva.

La proposición básica de este estudio es que si el gobierno saca mas ventaja política de los programas distributivos con respecto a la inversión para el crecimiento a largo plazo, éste se inclinará a asignar más fondos a gastos distributivos que desfavorablemente que afectan el crecimiento económico. Los resultados de la simulación están sobre todo consistentes con esta proposición. Los efectos de $\mu$ y $\eta$ en el crecimiento que arrojan los resultados de la simulación son bastante consistentes con nuestra propuesta teórica.

\section{CONCLUSIONES}

En este trabajo se examinó el impacto de las decisiones políticas del gobierno sobre el crecimiento económico en un marco endógeno con la estructura de la literatura existente y haciendo modificaciones en ella. Se demuestra cómo el gobierno determina la proporción de gasto público para maximizar su beneficio político y para examinar el efecto de las decisiones gubernamentales sobre el crecimiento económico. Se utilizó la estructura de los modelos teóricos existentes e hizo modificaciones en ella con objeto de estudiar la relación de la política distributiva con el crecimiento económico. El gobierno maximiza su interés político por la óptima elección de los instrumentos fiscales y la proporción de gasto público se determinó en función de los parámetros de ganancia política en un marco endógeno.

El modelo de Barro se amplió para incorporar dos tipos de gasto del gobierno: (i) la inversión para el crecimiento a largo plazo $\left(\mathrm{G}_{1}\right)$, y (ii) el gasto en políticas distributivas $\left(G_{2}\right)$. $G_{2}$ se supuso que es menos productivo que $G_{1}$. El resultado muestra que la asignación de más fondos para $\mathrm{G}_{2}$ reduce el crecimiento económico. Asimismo, el gobierno adopta medidas populistas a corto plazo y recurre a la política de distribución a fin de fortalecer su base de apoyo pero al mismo tiempo socava el crecimiento a largo plazo. No sólo los pobres, también los grupos acomodados de la sociedad, incluidos los grupos empresariales, grupos de presión de los agricultores, los empleados del gobierno y otros grupos de interés tratan de obtener beneficios inmediatos del gobierno en forma de subvenciones, sueldos, subsidios y concesiones. En efecto, el crecimiento de largo plazo se ve afectado.

El modelo de Agenor (2008) se incluye la ganancia política de $G_{2} . G_{2}$ entra en la función de utilidad de los hogares en forma no separable con el consumo privado. También entra en función de la producción y la función de la ganancia política del gobierno en el modelo modificado. Dos nuevos parámetros $\mu$ y $\eta$ que capturan la ganancia política de $G_{2}$ se introdujeron en el modelo. El resultado teórico propone que si la ganancia política de $G_{2}$ es más alta, el gobierno se 
inclina a asignar más fondos a las políticas distributivas y esto afecta negativamente el crecimiento económico. En la simulación del análisis numérico que se hizo para examinar los efectos de los dos nuevos parámetros $\mu$ y $\eta$; se encontró que si $\mu$ y $\eta$ aumentan, la tasa de crecimiento de estado de equilibrio disminuye. Los resultados encontrados en este documento contradice a los estudios Devarajan et al. (1996) y Ghosh y Gregoriou (2008) que encontraron que el gasto distributivo es más productivo que los gastos de capital. Sin embargo, nuestro resultados son congruentes con Chen (2006), Cristiano et al. (2009), García-Peñalosa y Turnovsky (2006) que encuentran que los gastos en infraestructura potencian el crecimiento pero no los gastos en programas distributivos.

\section{REFERENCIAS BIBLIOGRÁFICAS}

AGENOR, P. R. (2008). Fiscal policy and endogenous growth with public infrastructure, Oxford Economic Papers, 60, 57-87.

AIDT, T.S. \& SHVETS, J., (2011), Distributive Politics and Electoral Incentives: Evidence from Seven US State Legislatures, Cambridge Working Papers in Economics 1130, Faculty of Economics, University of Cambridge.

ALESINA, A., \& RODRICK, D. (1994). Redistributive Politics and Economic Growth. Quarterly Journal of Economics, 109, 465-90.

AFONSO, A. AND R. M. SOUSA (2009): "The Macroeconomic Effects of Fiscal Policy", ECB Working Paper Series, No. 991.

BANERJEE, A. V., \& NEWMAN, A. F. (1993). Occupational Choice and the Process of Development. Journal of Political Economy, 101(2), 274-98.

BARRO, R. J. (1990). Government Spending in a Simple Model of Endogenous Growth. Journal of Political Economy, 98(October) S103-S125.

BARRO, R. J., \& BECKER, G. S. (1989). Fertility choice in a Model of Economic Growth. Econometrica, 57(March), 481-501.

BARRO, R. J., \& SALA-I-MARTIN, X. (1995). Economic Growth. Boston: McGraw Hill.

CHEN, B. L. (2006). Economic Growth with an Optimal Public Spending Composition. Oxford Economic Papers, 58, 123-36.

CHRISTIANO, L., EICHENBAUM M. AND S. REBELO (2009): "When Is the Government Spending Multiplier Large?", NBER Working Paper, No. 15394.

DANIEL N. POSNER AND ERIC KRAMON (2011), Who Benefits from Distributive Politics? How the Outcome One Studies Affects the Answer One Gets, MIT Political Science Department Research Paper No. 2011-9. 
DEVARAJAN, S., SWAROOP, V., \& ZOU, H. (1996). The Composition of Public Expenditure and Economic Growth. Journal of Monetary Economics, 37, 313-344.

GALOR, O., \& ZEIRA, J. (1993). Income Distribution and Macroeconomics. Review of Economic Studies, 60(1), 35-52.

GALOR, O. (2011), Inequality and the Process of Development, Handbook of Economics of Education.

GARCÍA-PEÑALOSA, C., AND S.J. TURNOVSKY (2006), "Growth and income inequality: A canonical model." Economic Theory 28, 25-49.

GHOSH, S., \& GREGORIOU, A. (2008). The Composition of Government Spending and Growth: Is Current or Capital Spending Better? Oxford Economic Papers, 60, 484-516.

GROSSMAN, G. M. \& HELPMAN, E. (1991). Innovation and Growth in the Global Economy. Cambridge, MA: MIT Press.

MARJIT, S., KOLMAR, M., \& MUKHERJEE, V. (2001). Redistributive Politics, Corruption and Quality of Public Investment. Paper presented at the Fiscal Affairs Division, IMF.

MARJIT, S., \& MAITY, D. (2006). Politics and Contemporary Macroeconomy of India. In India Macroeconomics Annual, Centre for Studies in Social Sciences (pp. 9-55). India: Sage Publications Ltd.

MARJIT, S., MUKHERJEE, V., \& KOLMAR, M. (2006) Poverty, Taxation and Governance. Journal of International Trade \& Economic Development, 15(3), 325-333.

MELTZER, A. H., \& RICHARD, S. F. (1981). A Rational Theory of the Size of the Government. Journal of Political Economy, 89(51), 914-27.

PERSSON, T., \& TABELLINI, G. (1994). Is Inequality Harmful for Growth?. American Economic Review, 84(3), 600-621.

RAMSEY, F. (1928). A Mathematical Theory of Saving. Economic Journal, 38(December), 543-559.

REBELO, S. (1991). Long-run Policy Analysis and Long-run Growth. Journal of Political Economy, 99(June), 500-521.

ROMER, P. M. (1986). Increasing Returns and Long-run Growth. Journal of Political Economy, 94(October), 1002-1037.

ROMER, P. M. (1990). Endogenous Technological Change. Journal of Political Economy, 94(October), S71-S102.

SARKAR, A. (2006). Political Economy of West Bengal: A Puzzle and a Hypothesis. Economic and Political Weekly, 61(4), 341-48.

SASMAL, J. (2002). Size of Government, Nature of Government Spending and Level of Corruption in a Low Income Country: A Static Partial Equilibrium Analysis. Artha Beekshan, 11(3), 12-21.

SASMAL, J. (2004). Endogenous Growth with Government Spending - Effects of Size of Government, Distributive Politics and Corruption with Special Re- 
ference to Low Income Countries. Paper presented at the Annual Conference of the Dept. of Economics, Jadavpur University, India.

SASMAL, J. (2007). Size of Government, Distributive Politics and Governance : A Simple Political Choice Model. Proceedings of the 90th Annual Conference Volume, Indian Economic Association, 1240-1246.

UZAWA, H. (1965). Optimal technical change in an aggregative model of economic growth. International Economic Review, 6 January, 18-31. 


\section{Apéndice}

$$
\begin{gathered}
Y=A K^{\alpha} G_{1}^{\beta}\left(L G_{2}\right)^{1-\alpha-\beta} \\
y=A(k)^{\alpha}\left(g_{1}\right)^{\beta}\left(G_{2}\right)^{1-\alpha-\beta} \\
P M_{K}=\frac{\partial Y}{\partial K}=L \cdot A \cdot g_{1}^{\beta} \cdot G_{2}^{1-\alpha-\beta} \cdot a k^{\alpha-1} \cdot \frac{\partial k}{\partial K}=A g_{1}^{\beta} \cdot G_{2}^{1-\alpha-\beta} \cdot a k^{-(1-\alpha)} \\
G_{1}+G_{2}=G=(1-\psi) r \cdot Y \\
G_{1}=\lambda(1-\psi) r \cdot Y \quad \mathrm{y} \quad G_{2}=(1-\lambda)(1-\psi) r \cdot y \\
g_{1}=\frac{G_{1}}{L}=\frac{1}{L} \cdot \lambda(1-\psi) r \cdot Y
\end{gathered}
$$

Sustituyendo el valor de $Y$ de la ecuación (1) en $\mathrm{G}_{2}$, tenemos

$$
\begin{gathered}
G_{2}=(1-\lambda)(1-\psi) r \cdot A K^{\alpha} G_{1}^{\beta}\left(L G_{2}\right)^{1-\alpha-\beta} \\
\frac{G_{2}}{G_{2}^{1-\alpha-\beta}}=(1-\lambda)(1-\psi) r \cdot A K^{\alpha} G_{1}^{\beta} L^{1-\alpha-\beta} \\
\text { ó } \\
G_{2}=\left[(1-\lambda)(1-\psi) r \cdot A K^{\alpha} G_{1}^{\beta} G_{1}^{\beta}\right]^{\frac{1}{\alpha+\beta}}
\end{gathered}
$$

Después de simplificar, tenemos

$$
G_{2}=\{(1-\lambda)(1-\psi) r \cdot A\} \frac{1}{\alpha+\beta} k^{\frac{\alpha}{\alpha+\beta}} L^{\frac{1-\alpha-\beta}{\alpha+\beta}} G_{1}^{\frac{\beta}{\alpha+\beta}}
$$

De la misma manera, sustituyendo el valor de $Y$ en $G_{l}$, tenemos

$$
G_{2}=\lambda(1-\psi) r \cdot A K^{\alpha} G_{1}^{\beta}\left(L G_{2}\right)^{1-\alpha-\beta}
$$

Siguiendo el mismo procedimiento como en $\mathrm{G}_{2}$, tenemos

$$
\begin{aligned}
& G_{1}=\{\lambda(1-\psi) r \cdot A\} \frac{1}{\alpha-\beta} \cdot k^{\frac{\alpha}{1-\alpha}} \cdot L^{\frac{1-\beta}{1-\beta}} \cdot G_{2}{ }^{\frac{1-\alpha-\beta}{1-\beta}} \\
& g_{1}=\frac{G_{1}}{L}=\{\lambda(1-\psi) r \cdot A\} \frac{1}{1-\beta} \cdot k^{\frac{\alpha}{1-\beta}} \cdot G_{2}{ }^{\frac{1-\alpha-\beta}{1-\beta}}
\end{aligned}
$$

Ahora, sustituyendo el valor de $G_{2}$ de (2) en $G_{1}$, tenemos 
$G_{1}=\{\lambda(1-\psi) r \cdot A\} \frac{1}{1-\beta} \cdot k^{\frac{\alpha}{1-\beta}} \cdot L \cdot\left[\{(1-\lambda)(1-\psi) r \cdot A\}^{\frac{1}{\alpha+\beta}} \cdot k^{\frac{\alpha}{\alpha+\beta}} \cdot L^{\frac{1-\beta}{\alpha+\beta}}\right]^{\frac{1-\alpha-\beta}{1-\beta}} \cdot G_{1}^{\frac{\beta}{\alpha+\beta} * \frac{1-\alpha-\beta}{1-\beta}}$

Después de simplificar, $G_{l}$ se expresa como

$$
G_{1}=\lambda^{\frac{\alpha+\beta}{\alpha}}[(1-\psi) r A]^{\frac{1}{\alpha}}(1-\lambda)^{\frac{1-\alpha-\beta}{\alpha}} \cdot L^{\frac{1-\beta}{\alpha+\beta}} k
$$

Sustituyendo el valor de $G_{1}$ de (4) en $G_{2}$ de (2), tenemos

$$
G_{2}=\{(1-\lambda)(1-\psi) r A\} \frac{1}{\alpha+\beta} \cdot k^{\frac{\alpha}{\alpha+\beta}} L^{\frac{1-\beta}{\alpha+\beta}} \cdot\left\{\lambda^{\frac{\alpha+\beta}{\alpha}}[(1-\psi) r A]^{\frac{1}{\alpha}}(1-\lambda)^{\frac{1-\alpha-\beta}{\alpha}} \cdot L^{\frac{1-\beta}{\alpha}} \cdot k\right\}^{\frac{\beta}{\alpha+\beta}}
$$

Simplificando (5), tenemos

$$
G_{2}=\lambda^{\frac{\beta}{\alpha}} \cdot(1-\lambda)^{\frac{\alpha+\beta(1-\alpha-\beta)}{\alpha(\alpha+\beta)}} \cdot[(1-\psi) r A]^{\frac{1}{\alpha}} \cdot L^{\frac{1-\beta}{\alpha}} k
$$

Sustituyendo los valores de $\mathrm{g}_{1}$ y $G_{2}$ de (4) y (6) en $\mathrm{PM}_{\mathrm{K}}$, y simplificando, tenemos

$$
P M_{K}=\alpha A^{\frac{1}{\alpha}} \cdot \lambda^{\frac{\beta}{\alpha}} \cdot(1-\lambda)^{\frac{1-\alpha-\beta}{\alpha}}[(1-\psi) r]^{\frac{1-\alpha}{\alpha}} \cdot L^{\frac{\beta}{\alpha}}
$$

